\title{
Counting proofs in propositional logic
}

\author{
René DAVID* Marek ZAIONC ${ }^{\dagger}$
}

May 18, 2009

\begin{abstract}
We give a procedure for counting the number of different proofs of a formula in various sorts of propositional logic. This number is either an integer (that may be 0 if the formula is not provable) or infinite.
\end{abstract}

\section{Introduction}

The aim of the paper is to give a procedure for counting the number of different normal proofs of a formula in propositional logic. By the well known Curry Howard correspondence, this is similar to counting the number of different normal closed terms of some fixed type in an extension of the $\lambda \mu$ calculus.

We show that this number is the least fix-point of a system of polynomial equations in some natural complete lattice and we give an algorithm for finding such a least fix-point.

The similar problem of counting closed typed lambda terms was studied (see [1]) but never published by Ben- Yelles. Some description of the Ben-Yelles solution can be found in Hindley's book [4]. Similarly Hirokawa in [5] proved that the complexity of the question whether a given simple type (implicational formula) possess an infinite number of normal terms (or infinite number of proofs) is polynomial space complete. Recently similar research about counting $\lambda$-calculus objects for program synthesis was done by Wells and Yakobowski in [9].

\section{The logic}

\subsection{Formulae and proofs}

Definition 1 Let $\mathcal{A}$ be a set (possibly infinite) of atomic constants. The set $\mathcal{F}$ of formulae is defined by the following grammar

$$
\mathcal{F}::=\mathcal{A} \cup\{\perp\}|\mathcal{F} \rightarrow \mathcal{F}| \mathcal{F} \wedge \mathcal{F} \mid \mathcal{F} \vee \mathcal{F}
$$

We assume that $\perp \notin \mathcal{A}$ and, as usual, $\neg F$ will be an abbreviation for $F \rightarrow \perp$.

Definition 2 The rules for proofs in classical logic are the following.

\footnotetext{
*Lama, Université de Savoie, Campus Scientifique. 73376 Le Bourget du lac. Email : rene.david@univ-savoie.fr

†Theoretical Computer Science, Jagiellonian University, Lojasiewicza 6, 30-348 Kraków, Poland. Email : zaionc@tcs.uj.edu.pl. Research described in this paper is supported by Polish Ministry of Science and Higher Education grant NN206 356236
} 


$$
\begin{gathered}
\overline{\Gamma, A \vdash A} a x \\
\frac{\Gamma, A \vdash B}{\Gamma \vdash A \rightarrow B} \rightarrow \rightarrow_{i} \quad \frac{\Gamma_{1} \vdash A \rightarrow B \quad \Gamma_{2} \vdash A}{\Gamma_{1}, \Gamma_{2} \vdash B} \rightarrow_{e} \\
\frac{\Gamma_{1} \vdash A_{1} \quad \Gamma_{2} \vdash A_{2}}{\Gamma_{1}, \Gamma_{2} \vdash A_{1} \wedge A_{2}} \wedge_{i} \quad \frac{\Gamma \vdash A_{1} \wedge A_{2}}{\Gamma \vdash A_{i}} \wedge_{e} \\
\frac{\Gamma \vdash A_{j}}{\Gamma \vdash A_{1} \vee A_{2}} \vee_{i} \quad \frac{\Gamma \vdash A_{1} \vee A_{2}}{\Gamma_{1}, A_{1} \vdash C \quad \Gamma_{2}, A_{2} \vdash C} \\
\frac{\Gamma, \Gamma_{1}, \Gamma_{2} \vdash C}{\Gamma \vdash A \vdash \perp} \vee_{e} \\
\frac{\Gamma, \neg A \vdash A}{\Gamma, \neg A \vdash \perp} \perp_{i}
\end{gathered}
$$

\section{$2.2 \quad$ Terms coding proofs}

It is well known that a proof, in intuitionistic implicational logic, can be coded by a simply typed $\lambda$-term. The same thing can, in fact, be done for proofs, in classical logic, of any kind of formulae. The extension from intuitionistic logic to classical logic is the $\lambda \mu$-calculus introduced by Parigot in [6]. The extension to formulae using all the usual connectors has been introduced by de Groote in [3]. The next definition is a presentation of this calculus.

Definition 3 Let $\mathcal{V}$ and $\mathcal{W}$ be disjoint sets of variables. The set of $\lambda \mu^{\rightarrow \wedge}$-terms is defined by the following grammar

$$
\begin{gathered}
\mathcal{T}::=\mathcal{V}|\lambda \mathcal{V} \cdot \mathcal{T}|(\mathcal{T} \mathcal{E})|\langle\mathcal{T}, \mathcal{T}\rangle| \omega_{1} \mathcal{T}\left|\omega_{2} \mathcal{T}\right| \mu \mathcal{W} \cdot \mathcal{T} \mid(\mathcal{W} \mathcal{T}) \\
\mathcal{E}::=\mathcal{T}\left|\pi_{1}\right| \pi_{2} \mid[\mathcal{V} \cdot \mathcal{T}, \mathcal{V} \cdot \mathcal{T}]
\end{gathered}
$$

The next definition shows how the terms introduced in definition 3 code the proofs.

Definition 4 The typing rules for the $\lambda \mu \rightarrow \wedge \vee$-terms are as follows

$$
\begin{gathered}
\frac{\Gamma, x: A \vdash M: B}{\Gamma, x: A \vdash x: A} a x \quad \frac{\Gamma}{\Gamma \vdash \lambda x \cdot M: A \rightarrow B} \rightarrow_{i} \\
\frac{\Gamma_{1} \vdash M: A \rightarrow B \quad \Gamma_{2} \vdash N: A}{\Gamma_{1}, \Gamma_{2} \vdash(M N): B} \rightarrow_{e} \\
\frac{\Gamma, \alpha: \neg A \vdash M: A}{\Gamma, \alpha: \neg A \vdash(\alpha M): \perp} \perp_{i} \quad \frac{\Gamma, \alpha: \neg A \vdash M: \perp}{\Gamma \vdash \mu \alpha \cdot M: A} \perp_{e} \\
\frac{\Gamma_{1} \vdash M: A_{1} \quad \Gamma_{2} \vdash N: A_{2}}{\Gamma_{1}, \Gamma_{2} \vdash\langle M, N\rangle: A_{1} \wedge A_{2}} \wedge_{i} \quad \frac{\Gamma \vdash M: A_{1} \wedge A_{2}}{\Gamma \vdash\left(M \pi_{i}\right): A_{i}} \wedge_{e} \\
\frac{\Gamma \vdash M: A_{j}}{\Gamma \vdash \omega_{j} M: A_{1} \vee A_{2}} \vee_{i} \\
\frac{\Gamma \vdash M: A_{1} \vee A_{2} \quad \Gamma_{1}, x_{1}: A_{1} \vdash N_{1}: C \quad \Gamma_{2}, x_{2}: A_{2} \vdash N_{2}: C}{\Gamma, \Gamma_{1}, \Gamma_{2} \vdash\left(M\left[x_{1} \cdot N_{1}, x_{2} \cdot N_{2}\right]\right): C} \vee_{e}
\end{gathered}
$$

\section{Remark}

Note that, in definition 2, the letter $\Gamma$ represents a finite multi-set of formulae whereas, in definition 1 , it represents a finite multi-set of indexed formulae i.e. a finite set of pairs denoted as $x: A$ or $\alpha: \neg A$ where $x \in \mathcal{V}, \alpha \in \mathcal{W}$ and $A \in \mathcal{F}$ (where each variable occurs only once). 
In the rest of the paper, we will continue to use the same notation for these two formally distinct notions. Such a multi-set will be called a context. In a particular sentence which of the two notions is meant will usually be clear ... from the context.

Definition 5 The set $G$ of goals is the set of ordered pairs denoted as $\Gamma \vdash A$ where $A \in \mathcal{F}$ and $\Gamma$ is a context.

\subsection{Normal terms and proofs}

To avoid to have, for each formula, either zero or infinitely many proofs, we only consider proofs satisfying two conditions.

1. The first one is usual : we only look at normal proofs i.e. proofs with no cuts i.e. proofs such that the term that represents it is normal i.e. cannot be reduced by the reduction rules of definition 6 below which corresponds to the usual notion of cut elimination in natural deduction. Since every term is normalizing i.e. can be reduced to a normal term (cf. theorem \&, item 1), if a formula has a proof then it also has a normal proof. Thus the restriction does not change the problem.

2. The second restriction, though quite natural, is less usual but also necessary to avoid to have, for each formula, either zero or infinitely many proofs. It is as follows.

(a) When we are in one of the branch of a proof by case (we have $A \vee B$ as an hypothesis and we assume, for example, $A$ ), we are no more allowed to, again, distinguish the same two cases i.e. we delete the hypothesis $A \vee B$.

(b) We forbid to prove $\perp$ or $\neg C$ by contradiction.

(c) When we are in a part of the proof in which we already have assumed $\neg B$, toward a contradiction, we are no more allowed to prove $B$ by contradiction.

A proof satisfying these three conditions will be called fair. It is easy to check that if a formula is provable then it has a fair (normal) proof and thus asking for fairness does not change the problem. Since fairness is less usual, we will mention in the comments of section 3.4 where it appears in the research for proof.

Note finally that we may want to add some (optional) restrictions to the number of proofs we are looking for. They will be given in section 3.6 .

Definition 6 The reduction rules for the $\lambda \mu \rightarrow \wedge \vee$-calculus are given below. Variables $M, N, L$ are in $\mathcal{T}$ and $\varepsilon$ is in $\mathcal{E}$. A variable $x$ belongs to $\mathcal{V}$ while $\alpha$ is taken from $\mathcal{W}$.

$$
\begin{gathered}
(\lambda x \cdot M N) \triangleright_{\beta} M[x:=N] \\
\left(\left\langle M_{1}, M_{2}\right\rangle \pi_{i}\right) \triangleright M_{i} \\
\left(\omega_{i} M\left[x_{1} \cdot N_{1}, x_{2} \cdot N_{2}\right]\right) \triangleright N_{i}\left[x_{i}:=M\right] \\
\left(M\left[x_{1} \cdot N_{1}, x_{2} \cdot N_{2}\right] \varepsilon\right) \triangleright\left(M\left[x_{1} \cdot\left(N_{1} \varepsilon\right), x_{2} \cdot\left(N_{2} \varepsilon\right)\right]\right) \\
(\mu \alpha \cdot M \varepsilon) \triangleright_{\mu} \mu \alpha \cdot M[(\alpha L):=(\alpha(L \varepsilon))]
\end{gathered}
$$




\section{Remarks}

- The first three rules correspond to the elimination of a logical cut: an introduction rule is immediately followed by the corresponding elimination rule.

- The fourth rule corresponds to the so-called permutative conversion: if a case analysis is followed by an elimination rule the elimination can be done before the case analysis.

- The last rule corresponds to the so-called classical cuts

Note that the two last rules are necessary to ensure that a normal proof has the sub-formula property (cf. theorem 8, item 2).

Definition 7 Let $t$ be a $\lambda \mu^{\rightarrow \wedge \vee}$-term and $g=\Gamma \vdash A$ be a goal.

1. We say that $t$ is a proof of $g$ if $\Gamma \vdash t: A$.

2. We say that $t$ is normal if it contains no redex i.e. if it cannot be reduced by the rules of definition 6 .

Theorem 8 Let $t$ be a proof of $g=\Gamma \vdash A$. Then,

1. $t$ can be reduced into a normal proof of $g$.

2. If $t$ is normal and $B$ is a formula that occurs in the typing tree of then, there is a sub-formula $C$ of a formula in $\{A\} \cup \Gamma$ such that $B=C$ or $B=\neg C$.

Proof Standard. See for example [7] or [8].

Theorem 9 There is an algorithm that, given a formula $F$, computes the number (i.e. either an integer or $\infty$ ) of distinct normal and fair proofs of $F$.

Proof This is an immediate corollary of theorem 27 below whose statement and proof is given in the next section.

\section{Proof of the main result}

\subsection{The idea of the proof}

The idea of the proof is quite simple. To each goal $g$ of the form $\Gamma \vdash A$ we associate a variable $n_{g}$ that, intuitively, gives the number of normal and fair proofs of $g$. By looking at the possible ways of proving $g$ (either use an introduction rule or an elimination rule or a proof by contradiction) we get equations relating the $n_{g}$. We will show that the number we are looking for is the minimal solution of this set of equations. The two main technical difficulties are the following.

- We have to be able to compute the solution of these equations. This follows from the fact that they only use integers, addition and multiplication. An addition corresponds to the possibility of proving a goal in different ways. A multiplication means that, to prove the goal, we have to prove two different things. Thus the equations are polynomial and we will show that, for this kind of equations, we can always compute the minimal solution.

- The other point is a bit more difficult. To be able to compute its solution, the set of equations must be finite but, without sufficient care, it is not ! Since, by the sub-formula property (theorem 8 above), we know that the formulae that appear in a normal proof are sub-formulae of the initial formula, the set of goals must, intuitively, be finite (which would imply that the set of equations also is finite) but since, in $\Gamma$, a formula can be repeated many times it is not true that the set of goals is finite. To solve this problem, we proceed as follows. When, in a proof of some goal we introduce a new goal, say $h$, which is the same as a goal $h^{\prime}$ that has already 
been introduced except that it adds some hypothesis that were already present in $h^{\prime}$, we do not consider it as a new one i.e. we do not build an equation for it. This is because we can show that $h, h^{\prime}$ have the same number of proofs. But, to do that, we need some book keeping because to show that $h, h^{\prime}$ have the same number of normal and fair proofs, we need the fact that $h$ and $h^{\prime}$ are, somehow, in the same part of a proof. This will be ensured by the order we put on the variables $n_{g}$. Doing in this way, Konig's lemma ensures that the set of equations is finite.

\subsection{Polynomials}

\section{Definition 10 1. The set $\mathbb{N} \cup\{\omega\}$ will be denoted as $\overline{\mathbb{N}}$}

2. The usual order and operations on $\mathbb{N}$ are extended to $\overline{\mathbb{N}}$ by

- $i \leq \omega$ and $i+\omega=\omega+i=\omega$ for every $i \in \overline{\mathbb{N}}$,

- $0 \cdot \omega=\omega \cdot 0=0$,

- $i \cdot \omega=\omega \cdot i=\omega$ for every $i \neq 0$.

3. The set $\overline{\mathbb{N}}^{k}$ is naturally ordered by $\left(a_{1}, \ldots, a_{k}\right) \leq\left(b_{1}, \ldots, b_{k}\right)$ if $a_{i} \leq b_{i}$ for all $i$.

Lemma $11 \quad \overline{\mathbb{N}}^{k}$ is a complete lattice.

Proof Obvious.

Definition 12 1. The set of polynomials is the least set of functions (of several variables) from $\overline{\mathbb{N}}$ to $\overline{\mathbb{N}}$ that contains the constant functions and is closed by addition and multiplication.

2. The order on polynomials is the point-wise order, i.e. if $f\left(x_{1}, \ldots, x_{n}\right), g\left(x_{1}, \ldots, x_{n}\right)$ are polynomials, $f \leq g$ iff $\forall x_{1}, \ldots, x_{n}, f\left(x_{1}, \ldots, x_{n}\right) \leq g\left(x_{1}, \ldots, x_{n}\right)$.

Definition 13 1. A polynomial system of equations (PSE for short) is a set $\left\{E_{1}, \ldots, E_{n}\right\}$ where $E_{i}$ is the equation $x_{i}=f_{i}\left(x_{1}, \ldots, x_{n}\right)$ and $f_{i}$ is a polynomial in the variables $x_{1}, \ldots, x_{n}$. Such a system will be abbreviated as $\vec{x}=F(\vec{x})$.

2. Let $\vec{x}=F(\vec{x})$ by a PSE. We say that $\vec{a}$ is a minimal solution of the system if $\vec{a}=F(\vec{a})$ and, for every $\vec{b}$ such that $\vec{b}=F(\vec{b})$, we have $\vec{a} \leq \vec{b}$.

3. We denote by $F^{i}$ the i-iteration of $F$, i.e. $F^{0}(\vec{x})=\vec{x}$ and $F^{i+1}(\vec{x})=F\left(F^{i}(\vec{x})\right)$.

Proposition 14 Let $\vec{x}=F(\vec{x})$ be a PSE. Then, this system has a (unique) minimal solution $\vec{a}$ (that we will denote by $\min (F)$ ). Moreover we have $\min (F)=$ $\bigsqcup_{i=0}^{\infty} F^{i}(\overrightarrow{0})=\bigcap\{\vec{x} \mid F(\vec{x}) \leq \vec{x}\}$.

Proof Since it is easy to check that $F$ is increasing, this is a special case of the Knaster-Tarski lemma.

Lemma 15 Let $f(x, \vec{y})=f_{0}(\vec{y})+\sum_{i \geq 1} f_{i}(\vec{y}) x^{i}$ be a polynomial (where $\vec{y}$ is possibly empty) and let $h(\vec{y})=\sum_{i \geq 1} f_{i}(\vec{y})$. Then, $g(\vec{y})=f_{0}(\vec{y})+f_{0}(\vec{y}) \cdot h(\vec{y}) \cdot \omega$ is the minimal solution of the equation $x=f(x, \vec{y})$.

Proof If $f_{0}(\vec{y})=0$ then the minimal solution is 0 . If $h(\vec{y})=0$, then for all $i \geq 1$, $f_{i}(\vec{y})=0$ and the minimal solution is $f_{0}(\vec{y})$. Otherwise, it is easy to check that the minimal solution is $\omega$. In all cases the minimal solution is $g(\vec{y})$. 
Lemma 16 Let $\vec{x}=F(\vec{x})$ by a PSE. The minimal solution of this system can be computed from $F$.

Proof The algorithm to compute this solution is the following. Choose one variable, call it $x$ and call $\vec{y}$ the remaining variables. The system then looks like: $x=f(x, \vec{y})$ and $\vec{y}=G(x, \vec{y})$. Use lemma 15 to find the polynomial $g(\vec{y})$ which is the minimal solution of the equation $x=f(x, \vec{y})$. Repeat the process with the system $\vec{y}=G(g(\vec{y}), \vec{y})$. It is clear that, in this way, we find a solution of the system. Denote by $(a, \vec{b})$ this solution. By proposition 14 , let $\left(x_{0}, \overrightarrow{y_{0}}\right)=\min (F)$. Since $(a, \vec{b})$ is a solution of the system we have $\left(x_{0}, \overrightarrow{y_{0}}\right) \leq(a, \vec{b})$. Thus it remains to show that $(a, \vec{b}) \leq\left(x_{0}, \overrightarrow{y_{0}}\right)$. Since $x_{0}$ is a solution of the equation $x=f\left(x, \overrightarrow{y_{0}}\right)$ we have $g\left(\overrightarrow{y_{0}}\right) \leq x_{0}$. Define $F^{\prime}$ by $F^{\prime}(\vec{y})=G(g(\vec{y}), \vec{y})$. By the monotonicity of $G$, $F^{\prime}\left(\vec{y}_{0}\right)=G\left(g\left(\vec{y}_{0}\right), \vec{y}_{0}\right) \leq G\left(x_{0}, \vec{y}_{0}\right)=\vec{y}_{0}$. But since the minimal solution of $F^{\prime}$ is $\bigcap\left\{\vec{y} \mid F^{\prime}(\vec{y}) \leq \vec{y}\right\}$ we have $\vec{b} \leq \overrightarrow{y_{0}}$. By the monotonicity of $g, a=g\left(b_{0}\right) \leq g\left(\vec{y}_{0}\right) \leq x_{0}$.

\subsection{Some preliminary results}

Definition $17 \quad$ 1. We will denote by $\mathcal{F}^{\prime}$ the set of formulae to which we have added a special element denoted as $*$.

2. Let $E$ be a set of lists of elements of $\mathcal{F}^{\prime}$ and $A$ be a formula. We will denote by $[A:: E]$ the set $\{[A:: L] \mid L \in E\}$ where $[A:: L]$ denotes the list $L$ on the beginning of which we have added $A$.

\section{Remark}

Note that the definition implies that, if $E$ is empty, then so is $[A:: E]$.

Definition 18 Let $A, B$ be formulae. The set $\operatorname{Elim}(A, B)$ of lists of elements of $\mathcal{F}^{\prime}$ is defined, by induction on the size of $A$, in the following way.

1. If $A=B$, then $\operatorname{Elim}(A, B)=[*]$.

2. If $A \neq B$ then,

- If $A$ is atomic, $\operatorname{Elim}(A, B)=\emptyset$

- If $A=C \rightarrow D, \operatorname{Elim}(A, B)=[C:: \operatorname{Elim}(D, B)]$

- If $A=A_{1} \wedge A_{2}, \operatorname{Elim}(A, B)=\operatorname{Elim}\left(A_{1}, B\right) \cup \operatorname{Elim}\left(A_{2}, B\right)$

- If $A=A_{1} \vee A_{2}, \operatorname{Elim}(A, B)=\{[A]\}$

Lemma 19 Let $A, B$ be formulae and let $L \in \operatorname{Elim}(A, B)$. Then the last element of $L$ is either * or a disjunction.

Proof By induction on $A$.

\section{Comments and examples}

1. The role of the particular symbol $*$ and the set $\operatorname{Elim}(A, B)$ will become clear in item 3 of the next lemma. The intuition is the following. $\operatorname{Elim}(A, B)$ is the set of lists $L$ satisfying the following properties.

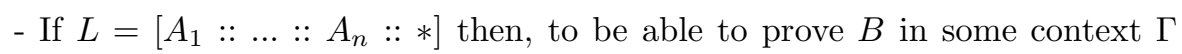
by using a sequence of elimination rules starting with $A$, it is enough to prove $A_{1}, \ldots, A_{n}$ in the context $\Gamma$.

- If $L=\left[\begin{array}{llllll}A_{1}:: \ldots & :: A_{n-1}:: D_{1} \vee D_{2}\end{array}\right]$ then, to be able to prove $B$ in some context $\Gamma$ by using a sequence of elimination rules starting with $A$, it is enough to prove $A_{1}, \ldots, A_{n-1}$ in the context $\Gamma$ and to prove $B$ both in the contexts $\Gamma \cup\left\{D_{1}\right\}$ and $\Gamma \cup\left\{D_{2}\right\}$. 
2. Assume $B, B^{\prime}$ are distinct atomic formulae and $A=\left(A_{1} \rightarrow D_{1} \vee D_{2}\right) \wedge\left(A_{2} \rightarrow\right.$ $\left.A_{3} \rightarrow B\right) \wedge\left(A_{4} \rightarrow B^{\prime}\right)$. Then $\operatorname{Elim}(A, B)=\left\{L_{1}, L_{2}\right\}$ where $L_{1}=\left[A_{1}::\right.$ $\left.D_{1} \vee D_{2}\right]$ and $L_{2}=\left[A_{2}:: A_{3}:: *\right]$

Lemma 20 Let $t$ be a normal proof of $\Gamma \vdash B$. Then, $t$ is in one of the following form (where the $t_{i}$ are normal)

\section{Either}

- $t=\lambda x \cdot t_{1}, B=B_{1} \rightarrow B_{2}$ and $\Gamma, x: B_{1} \vdash t_{1}: B_{2}$

- $t=\mu \alpha . t_{1}$ and $\Gamma, \alpha: \neg B \vdash t_{1}: \perp$

- $t=\left\langle t_{1}, t_{2}\right\rangle, B=B_{1} \wedge B_{2}$ and $\Gamma \vdash t_{i}: B_{i}$

- $t=\omega_{i} t_{1}, B=B_{1} \vee B_{2}$ and $\Gamma \vdash t_{1}: B_{i}$.

2. Ort $t=\left(\alpha t_{1}\right)$ and $\Gamma \vdash t_{1}: A$ where $\Gamma \vdash \alpha: \neg A$

3. Or $t=\left(\begin{array}{llll}x & t_{1} & \ldots & t_{n}\end{array}\right)$ and, for some $A$ such that $\Gamma \vdash x: A$ and some $L \in$ $\operatorname{Elim}(A, B)$, we have

- either $L=\left[A_{1}:: \ldots:: A_{n}:: *\right]$ and the $t_{i}$ are proofs of $\Gamma \vdash A_{i}$

- or $L=\left[A_{1}:: \ldots: A_{n-1}:: D_{1} \vee D_{2}\right]$ and, for $i<n$, the $t_{i}$ are proofs of $A_{i}$ and $t_{n}=\left[x_{1} \cdot u_{1}, x_{2} \cdot u_{2}\right]$ and the $u_{i}$ are proofs of $\Gamma, x_{i}: D_{i} \vdash B$.

Proof By induction on the size of the proof. The only non immediate point is that we cannot use an elimination rule when the type is a disjunction. This is because, otherwise, we will get a proof of the form $\left(\begin{array}{llll}x & t_{1} & \ldots & t_{k}\end{array}\left[x_{1} \cdot N_{1}, x_{2} . N_{2}\right] \varepsilon\right)$ which is not normal.

Definition 21 Let $t$ be a normal proof. The size of $t$ (denoted as size $(t)$ ) is defined as follows.

1. $\operatorname{size}\left(\lambda x . t_{1}\right)=\operatorname{size}\left(\mu \alpha . t_{1}\right)=\operatorname{size}\left(\omega_{i} t_{1}\right)=\operatorname{size}\left(t_{1}\right)+1$

2. $\operatorname{size}\left(\left\langle t_{1}, t_{2}\right\rangle\right)=\max \left(\operatorname{size}\left(t_{1}\right)\right.$, size $\left.\left(t_{2}\right)\right)+1$

3. $\operatorname{size}\left(\left(x t_{1} \ldots t_{n}\right)=\max \left(\operatorname{size}\left(t_{1}\right), \ldots\right.\right.$, size $\left.\left(t_{n}\right)\right)+1$

Definition 22 1. The set $\mathcal{P}$ of partial (normal) terms is defined by the following grammar

$$
\mathcal{P}:=\mathcal{V}|G| \lambda x . \mathcal{P}|\mu \alpha . \mathcal{P}|\langle\mathcal{P}, \mathcal{P}\rangle\left|\omega_{i} \mathcal{P}\right|(x \mathcal{P} \ldots \mathcal{P})
$$

2. The typing rules for $\mathcal{P}$ are the ones of $\mathcal{T}$ plus the additional rule

$$
\overline{\Gamma \vdash g: A} \quad \text { if } g=\Gamma \vdash A
$$

\section{Remark}

A normal proof is partial term that contains no goal.

Definition 23 Let $g$ be a goal. We denote by \# $(g)$ the number (considered as an element of $\overline{\mathbb{N}}$ ) of distinct normal and fair proofs of $g$.

Definition 24 1. Let $\Gamma, \Gamma^{\prime}$ be two contexts. We say that $\Gamma$ is equivalent to $\Gamma^{\prime}$ (denoted as $\Gamma \sim \Gamma^{\prime}$ ) if, for any $A \in \mathcal{F}, \Gamma$ contains a declaration $x: A$ iff $\Gamma^{\prime}$ contains a declaration $y: A$.

2. Let $g=\Gamma \vdash B$ and $g^{\prime}=\Gamma^{\prime} \vdash B^{\prime}$. We say that $g$ is equivalent to $g^{\prime}$ (denoted as $\left.g \sim g^{\prime}\right)$ if $B=B^{\prime}$ and $\Gamma \sim \Gamma^{\prime}$. 
Thus two goals $g, g^{\prime}$ are equivalent iff their conclusions are the same and they have same set of hypothesis but each hypothesis may appear a different number of times in $g$ and $g^{\prime}$.

Lemma 25 Let $t$ be a partial proof of goal $g$. Assume $t \neq g$ and contains some goal $g^{\prime} \sim g$. Then $\#(g)=\#\left(g^{\prime}\right)$.

Proof It is clear that $g$ has no proof iff $g^{\prime}$ has no proof. Assume then that $\#(g) \geq 1$. Let $g^{\prime \prime}=\Gamma^{\prime \prime} \vdash A \sim g$ be such that, for any formula $B$, the number of occurrences of $B$ in $\Gamma$ or in $\Gamma^{\prime}$ is less or equal to the number of occurrences of $B$ in $\Gamma^{\prime \prime}$.

We first show that $\#\left(g^{\prime \prime}\right)=\omega$. It is clear that the term $t^{\prime}$ obtained from $t$ by replacing $g^{\prime}$ by $g^{\prime \prime}$ also is a partial proof of $g^{\prime \prime}$ and, if $u$ is a proof of $g$, it also is a proof of $g^{\prime \prime}$. Then, the $u_{n}$ defined by $u_{0}=u$ and $u_{n+1}=t^{\prime}\left[g^{\prime \prime}:=u_{n}\right]$ are distinct normal and fair proofs of $g$.

We then show that $\#(g)=\omega$ (and, by symmetry, \#( $\left.g^{\prime}\right)=\omega$ ). Assume, toward a contradiction, that $\#(g)$ is finite. To each proof of $g^{\prime \prime}$ associate the proof of $g$ obtained by replacing the occurrences of a variable in $\Gamma^{\prime \prime}-\Gamma$ by one with the same type in $\Gamma$. Since \# $(g)$ is finite and \# $\left(g^{\prime \prime}\right)$ is infinite, there are infinitely many proofs of $g^{\prime \prime}$ that have the same image by this transformation. But this is impossible since, in a proof, each variable occurs only finitely many times.

\subsection{The equations}

To every goal $g=\Gamma \vdash A$ we associate a polynomial system of equations (denoted as $P S E(g))$ of the form $\vec{n}=P(\vec{n})$ where a goal $g_{i}$ is associated to each variable $n_{i}$ and $p_{i}$ is a polynomial that, intuitively, computes the number of normal and fair proofs of $g_{i}$ of a given size from the number of proofs (of smaller size) of the other goals needed to prove $g_{i}$.

$P S E(g)$ is defined by the following algorithm. This algorithm builds, step by step, a partially ordered set $V$ of variables (denoted as $n$ with some index), a function $F$ that associates goals to the variables and a set $E$ of equations of the form $n_{i}=p_{i}(\vec{n})$. We will show (see lemma 26 below) that it terminates. $P S E(g)$ will be the set of equations we have built when the algorithm terminates.

It is important to note that the function $F$ is not necessarily injective i.e. to different variables may correspond to the same goal. The reason will be given in the comments after the description of the algorithm.

- Initial step

Set $V=\left\{n_{0}\right\}, F\left(n_{0}\right)=g$ and $E=\emptyset$.

- General step

If, for all $n_{i} \in V$, there is an equation $n_{i}=p_{i}(\vec{n})$ in $E$, then stop. Otherwise, choose some $n_{i}$ for which $E$ has no equation. We introduce new variables and build the polynomial $p_{i}$ as the sum of three polynomials in the following way. The first one corresponds to a proof of $F\left(n_{i}\right)=\Gamma \vdash B$ beginning by an introduction rule, the second corresponds to a proof of $F\left(n_{i}\right)$ by contradiction and the last corresponds to a proof of $F\left(n_{i}\right)$ by using some hypothesis and several elimination rules.

In the definition of these polynomials we will adopt the following convention. If $h$ is a goal, when we say " let $n$ be a variable for $h$ " (we will also say " $n$ is a name for $h$ ") this will mean that either $F\left(n_{j}\right) \sim h$ for some $n_{j}<n_{i}$ and then $n$ is such an $n_{j}$ (if there are several choose one) or, if no such variable exists, choose a fresh index $j$ and set $F\left(n_{j}\right)=h$. For each variable $n_{j}$ introduced in this way, we set $n_{j}>n_{k}$ for each $k$ such that $n_{i} \geq n_{k}$. 
1. The first polynomial $P$ depends on the main connector of $B$.

(a) If $B$ is an atomic formula, then $P=0$

(b) If $B=C \rightarrow D$ then let $h=\Gamma, y: C \vdash D$, then let $P=n_{j}$ where $n_{j}$ is a variable for $h$.

(c) If $B=B_{1} \wedge B_{2}$. Let $h_{i}$ be the goal $\Gamma \vdash B_{i}$. Then $P=n_{i_{1}} \cdot n_{i_{2}}$ where $n_{i_{1}}, n_{i_{2}}$ are variables for $h_{1}, h_{2}$.

(d) If $B=B_{1} \vee B_{2}$. Let $h_{i}$ be the goal $\Gamma \vdash B_{i}$. Then $P=n_{i_{1}}+n_{i_{2}}$ where $n_{i_{1}}, n_{i_{2}}$ are variables for $h_{1}, h_{2}$.

2. The second polynomial $Q$ is as follows.

(a) If $B=\perp$ or $B=\neg C$ or if there is already in $\Gamma$ an hypothesis of the form $\alpha: \neg B$, then $Q=0$.

(b) Otherwise, let $h=\Gamma, \alpha: \neg B \vdash \perp$ and $Q=n_{j}$ where $n_{j}$ is a variable for $h$.

3. The last polynomial is the sum of (over all the hypothesis $H$ in $\Gamma$ ) of the polynomials $R_{H}$ defined as follows.

(a) If $H$ is $x: A, R_{H}$ is the sum (over $L \in \operatorname{Elim}(A, B)$ ) of the polynomials $R_{H, L}$ defined below.

- Assume $L=\left[A_{1}:: \ldots .: A_{p}:: *\right]$. Then $R_{H, L}=n_{i_{1}} \ldots . n_{i_{p}}$ where $g_{i}=\Gamma \vdash A_{i}$ and $n_{i_{1}}, \ldots, n_{i_{p}}$ are variables for $g_{1}, \ldots, g_{p}$. In particular, if $p=0$, this means $R_{H, L}=1$.

- Assume $L=\left[\begin{array}{lllllll}A_{1} & :: & \ldots & :: A_{p}:: D_{1} \vee D_{2}\end{array}\right]$. Then, let $g_{i}=\Gamma \vdash A_{i}$, $h_{i}=\Gamma^{\prime}, y: D_{i} \vdash B$ where $\Gamma^{\prime}$ is $\Gamma$ from which we have deleted the hypothesis $x$ : $A$. Let $n_{i_{1}}, \ldots, n_{i_{p}}$ be variables for $g_{1}, \ldots, g_{p}$, let $n_{j_{1}}, n_{j_{2}}$ be variables for $h_{1}, h_{2}$. Then $R_{H, L}=n_{i_{1}} . \ldots . n_{i_{p}} . n_{j_{1}} . n_{j_{2}}$

(b) If $H$ is $\alpha: \neg A$ then $R_{H}=n_{j}$ where $h=\Gamma \vdash A$ and $n_{j}$ is a variable for $h$.

\section{Comments}

1. Eliminating the hypothesis $x: A$ in the case of an elimination of the disjunction is condition (a) of fairness. The fact that $Q=0$ in the first case of a proof by contradiction is condition (b) and (c) of fairness.

2. The fact that a goal may have different names i.e. we may have $F\left(n_{i}\right)=F\left(n_{j}\right)$ for $i \neq j$ comes from the following reason. A goal $h$ may appear in different proofs of $g$ or in different parts of a proof of $g$. Of course $\#(h)$ does not depend on the place where $h$ appears but the condition that lets us decide to give it a new name or not depends of this place. We know, by lemma 25, that $\#(h)=\#\left(h^{\prime}\right)$ if $h \sim h^{\prime}$ and $h$ is below $h^{\prime}$ in some part of a proof but there is no reason to have \# $(h)=\#\left(h^{\prime}\right)$ if they appear in different proofs or in independent part of a proof.

Lemma 26 The algorithm given above terminates.

Proof Since the goals are made of sub-formulae of the formulae in $g$, there are only finitely many possible non equivalent goals. Also note that, when we try to find a proof for a goal $h$ and we have to consider some goal $h_{1}$, we give a new name to $h_{1}$ (i.e. we introduce a new variable $n_{i}$ such that $F\left(n_{i}\right)=h_{1}$ for which, later, we will have to find an equation) only when there is no $h_{2} \sim h_{1}$ below $h$ in the branch of the proof of $g$ that the algorithm, intuitively, constructs. Thus, all the branches are finite. Since there are only finitely many rules, by Konig's lemma, only finitely many variables can be introduced and thus the algorithm terminates. 


\subsection{Proof of theorem 9}

It is an immediate consequence of lemma 25 and theorem 27 below.

Theorem 27 Let $g$ be a goal and let $\vec{a}$ be the minimal solution of PSE $(g)$. Then, for each variable $n_{i}$ occurring in $P S E(g)$ we have $a_{i}=\#\left(F\left(a_{i}\right)\right)$.

Proof Let $P S E(g)$ be the set $\vec{n}=P(\vec{n})$ of equations and $\vec{b}$ be defined by $b_{i}=$ $\#\left(F\left(n_{i}\right)\right)$. It follows from lemma 25 that $\vec{b}$ is a solution of $P S E(g)$. Thus, we have $\vec{a} \leq \vec{b}$. Let $u_{k}=P^{k}(\overrightarrow{0})$. Since $\vec{a}$ is the minimal solution of the system $\vec{n}=P(\vec{n})$ we have $\vec{a}=\bigsqcup_{k=0}^{\infty} u_{k}$. Denote by $d_{i}(k)$ the number of normal and fair proofs of $F\left(n_{i}\right)$ of size $k$ and $\overrightarrow{d(k)}$ the vector whose components are the $d_{i}(k)$. Then $\vec{b}=\bigsqcup_{k=0}^{\infty} \overrightarrow{d(k)}$. Note that the equations are done so that $\overrightarrow{d(k+1)} \leq P(\overrightarrow{d(k)})$. An immediate induction shows that, for each $k, \overrightarrow{d(k)} \leq u_{k}$. It follows then that $\vec{b} \leq \vec{a}$.

\section{Remark}

If, instead of interpreting the variables and coefficients in $\overline{\mathbb{N}}$, we interpret them in the set $\{0,1\}$ where the operations and the order are the ones of $\mathbb{N}$ except that $1+1=1$, the conclusion of the theorem is then that $a_{h}=1$ iff the goal $h$ is provable.

\subsection{Some other restrictions on proofs}

Definition 28 We say that a normal term $t$ is in $\eta$-long normal form if the following holds for every sub-term $u$ of $t$.

1. If $u$ has type $A \rightarrow B$ then either $u=\lambda x . u^{\prime}$ or $u$ is applied to some other term.

2. If $u$ has type $A \wedge B$ then $u=\left\langle u_{1}, u_{2}\right\rangle$ for some terms $u_{1}, u_{2}$.

The algorithm we have given in the previous sections has been designed to get the number of normal and fair proofs in classical logic. It can be easily transformed if we want to only count proofs satisfying some constraints.

1. If we want to have proofs in minimal logic i.e. the logic where the rules $\perp_{i}$ and $\perp_{e}$ are deleted, we just forget the second step (which corresponds to proof by contradiction) in the definition of the set of equations

2. If we want to have proofs in intuitionistic logic, i.e. the logic where the rules $\perp_{i}$, and $\perp_{e}$ are deleted and replaced by the rule

$$
\frac{\Gamma \vdash \perp}{\Gamma \vdash A}
$$

we replace the polynomial given in the second step of the definition of the set of equations by the following one. If $g$ is $\Gamma \vdash B$ and $B \neq \perp$ then $Q=n_{h}$ where $h$ is $\Gamma \vdash \perp$ and $Q=0$ otherwise.

3. Instead of changing the logic, we may also want to restrict the form of the proofs we are looking for. The main usual restriction is to ask to have proofs in $\eta$-long normal form. It is well known that, with this restriction, the system remains complete. If we want such proofs it is enough, in the definition of the equations to ask that, if the goal is $\Gamma \vdash B$ and the main connector of $B$ is either an arrow or a conjunction, then we cannot use a proof by contradiction or use an elimination rule.

4. Our algorithm gives two normal and fair proofs for the formula $A \rightarrow A$. These proofs are $\lambda x . x$ and $\lambda x . \mu \alpha .(\alpha x)$. We could consider that these two proofs are the same and, actually, there is a reduction rule in the $\lambda \mu$-calculus that 
ensures that the second term reduces to the first one. This rule, that looks like the $\eta$-rule of the $\lambda$-calculus, is the following $\mu \alpha .(\alpha M) \triangleright M$ if $\alpha$ does not occur in $M$. It intuitively means that if, in a proof of $A$ by contradiction, in fact you have a proof $M$ of $A$ that does not use $\neg A$, you can eliminate the use of the rule for proof by contradiction.

It would be more difficult to consider this rule in the definition of normal proof. This is because it is non local and our algorithm, by essence, can only consider local configurations.

\subsection{From polynomials to formulae}

In the previous sections we have associated to each formula $F$ a set of polynomial equations whose minimal solution gives the number of normal and fair proofs of $F$. The opposite construction is also possible as the next proposition shows.

Definition 29 Let $F$ be a formula of implicational propositional logic i.e. $F$ is built from atomic formulae by using only the arrow as connectors. The rank of $F$ (denoted as $r(F)$ ) is defined by the following rules.

1. If $F$ is atomic, then $r(F)=0$

2. If $F=A \rightarrow B$, then $r(F)=\max (r(A)+1, r(B))$

Proposition 30 Let $E$ be a polynomial system of equations with $n$ variables. We can compute $n$ formulae $A_{1}, \ldots, A_{n}$ of implicational logic such that, if $\left(a_{1}, \ldots, a_{n}\right)$ is the minimal solution of $E$ then, for all $i \leq n, a_{i}$ is the number of proofs of $A_{i}$ in $\eta$-long normal form. Moreover we may assume that $r\left(A_{i}\right) \leq 2$ for all $i \leq n$.

Proof Let $\vec{x}=F(\vec{x})$ be the system and $F=\left(f_{1}, \ldots, f_{n}\right)$. We take $n$ fresh ground types $O_{1}, \ldots, O_{n}$. For each polynomial $f_{p}$ we construct a formula $B_{p}$ in the following way. For each monomial $M_{i}=x_{1}^{\alpha_{1}} \cdot \ldots \cdot x_{n}^{\alpha_{n}}$ which appears in $f_{p}$ let $T_{i}$ be the formula $O_{1}^{\alpha_{1}}, \ldots, O_{n}^{\alpha_{n}} \rightarrow O_{p}$. Remember that constant 1 can be obtained as the monomial $x_{1}^{\alpha_{1}} \cdot \ldots \cdot x_{n}^{\alpha_{n}}$ when all $\alpha_{i}=0$. The formula associated to $f_{p}$ is $T_{1}, \ldots, T_{m} \rightarrow O_{p}$. The fact that these formulae satisfy the desired conclusion is straightforward.

\section{Examples}

\section{Example 1} below

We want to compute the number of normal and fair proofs of the formula $F$

$$
F=F_{1} \rightarrow F_{2} \rightarrow F_{3} \rightarrow F_{4} \rightarrow F_{5} \rightarrow F_{6} \rightarrow A
$$

where

$$
\begin{array}{ll}
F_{1}=B \rightarrow C \rightarrow C \\
F_{5}=C \rightarrow C \rightarrow A
\end{array} \quad \begin{aligned}
& F_{2}=F_{3}=C \\
& F_{6}=A \rightarrow B \rightarrow A
\end{aligned} \quad F_{4}=B \rightarrow C \rightarrow B
$$

To avoid too many equations we will restrict ourselves to proofs in $\eta$-long normal form and in minimal logic and, to simplify notations, we will use the same name for a goal and the variable attached to it and, if a goal has several names, the corresponding variables will be the same with, possibly, some index. Also note that, since we will not write the terms representing the proofs, there is no need to give names to the hypothesis and thus we will write contexts simply as multi-sets of formulae.

Let $\Gamma=F_{1}, F_{2}, F_{3}, F_{4}, F_{5}, F_{6}$. The goals are: 


$$
\begin{gathered}
x \text { is } \Gamma \vdash A, \\
y, y_{1} \text { are } \Gamma \vdash B \\
z, z_{1} \text { are } \Gamma \vdash C .
\end{gathered}
$$

The order on these variables is given by: $x<y, z ; y<z_{1}$ and $z<y_{1}$.

The set of equations is

$$
\begin{array}{lr}
r=x y+z^{2} & \\
y=y z_{1} & z_{1}=2+y z_{1} \\
z=2+y_{1} z & y_{1}=y_{1} z
\end{array}
$$

The minimal solution is $x=4, y=y_{1}=0, z=z_{1}=2$ and, therefore, there are exactly 4 proofs of $F$ in $\eta$-long normal form.

\section{Example 2}

We want to compute the number of normal and fair proofs of the formula $F$ below where $\neg_{c} B$ is the abbreviation of $B \rightarrow C$. This formula is a kind of translation (provable in minimal logic) of Pierce law.

$$
F=\left(\left(A \rightarrow \neg \neg_{c} \neg_{c} B\right) \rightarrow \neg_{c} \neg_{c} A\right) \rightarrow \neg_{c} \neg_{c} A
$$

Again, we adopt the same restrictions and conventions of notations as in the previous example.

Let $F_{1}=\left(A \rightarrow \neg_{c} \neg_{c} B\right) \rightarrow \neg_{c} \neg_{c} A, F_{2}=\neg_{c} A$ and $\Gamma=\alpha_{1}: F_{1}, \alpha_{2}: F_{2}$.

The goals are

$$
\begin{gathered}
x \text { is } \Gamma \vdash C, \\
y, y_{1} \text { are } \Gamma, A, \neg_{c} B \vdash C, \\
z, z_{1} \text { are } \Gamma, A \vdash C, \\
u \text { is } \Gamma \vdash A, \\
v, v_{1} \text { are } \Gamma, A, \neg{ }_{c} B \vdash A, \\
w, w_{1} \text { are } \Gamma, A, \neg_{c} B \vdash B \\
r, r_{1} \text { are } \Gamma, A \vdash A .
\end{gathered}
$$

The order on these variables is given by: $x<y, z, u ; y<z_{1}, v, w ; z_{1}<r_{1}$; $z<y_{1}, r ; y_{1}<v_{1}, w_{1}$

The set of equations is

$$
\begin{aligned}
& x=y z+u \\
& y=y z_{1}+v+w \quad z_{1}=y z_{1}+r_{1} \\
& z=y_{1} z+r \quad y_{1}=y_{1} z+v_{1}+w_{1} \\
& v=v_{1}=1 \quad w=w_{1}=0 \quad r=r_{1}=1 \quad u=0
\end{aligned}
$$

The minimal solution is $x=y=z=y_{1}=z_{1}=\omega$ and, therefore, there are infinitely many proofs of $F$ in $\eta$-long normal forms.

\section{Example 3}

Let $F$ be the formula $\neg A \vee A$. It is known that $F$ is not provable in intuitionistic logic. We will show that, in classical logic, the are infinitely many distinct proofs in $\eta$-long normal form. Since the number of equations to be written is quite big we will only write some of those that imply that the number is infinite. To simplify we will also omit some intermediate goals and/or equations when the relations between the corresponding variables are easy to show.

The useful goals are the following 


$$
\begin{gathered}
x \text { is } \vdash F \\
x_{1} \text { is } \vdash A, x_{2} \text { is } \vdash \neg A \text { and } x_{3} \text { is } \alpha: \neg F \vdash \perp \\
a \text { is } \alpha: \neg F \vdash A \text { and } b \text { is } \alpha: \neg F \vdash \neg A \\
a_{1} \text { is } \alpha: \neg F, \beta: \neg A \vdash \perp \text { and } a_{2} \text { is } \alpha: \neg F \vdash \neg A \\
c \text { is } \alpha: \neg F, \beta: \neg A, y: A \vdash \perp \\
c_{1} \text { is } \alpha: \neg F, \beta: \neg A, y: A \vdash A \\
d \text { is } \alpha: \neg F, \beta: \neg A, y: A, z: A \vdash \perp
\end{gathered}
$$

Some equations are

$$
\begin{gathered}
x=x_{1}+x_{2}+x_{3} \\
x_{1}=0, x_{2}=0 \\
x_{3}=a+b \\
a=a_{1}+a_{2} \\
a_{1}=c \quad(\star) \\
c=2 . c_{1}+d \\
c_{1}=1
\end{gathered}
$$

The use of lemma 25 gives $d=c$.

( $a_{1}$ actually is the sum of $c$ and some other variables that are easily shown to be 0 .

\section{References}

[1] C.B Ben-Yelles. Type assignment in the lambda calculus. Syntax and semantics. Thesis, Mathematics Department, University of Wales Swansea, Swansea, UK (1979).

[2] W. Dekkers. Reducibility of types in Typed Lambda Calculus. Information and Computation vol 77, No 2 pp 131-137 (1988).

[3] P. de Groote. Strong Normalization of Classical Natural Deduction with Disjunction. Springer Lecture Notes in Computer Science 2044 pp 182-196 (2001).

[4] J.R. Hindley. Basic Simple Type Theory. Cambridge Tracts in Theoretical Computer Science 42. Cambridge University Press 1997.

[5] S. Hirokawa. Infiniteness of Proof $(\alpha)$ is P-Space Complete. Theoret. Comput. Sci. 206 no. 1-2, pp 331-339 (1998).

[6] M. Parigot. $\lambda \mu$-Calculus: An Algorithmic Interpretation of Classical Natural Deduction. Springer Lecture Notes in Computer Science 624 pp 190-201 (1992).

[7] A.S. Troelstra, H. Schwichtenberg. Basic proof theory. Cambridge University Press 1996.

[8] D. Van Dalen. Logic and structure. Springer 1997.

[9] J. B. Wells, B. Yakobowski. GraphBased Proof Counting and Enumeration with Applications for Program Fragment Synthesis. Springer Lecture Notes in Computer Science 3573, pp 262-277 (2005). 